Running Head: Statistical learning and information weights

\title{
Age-dependent statistical learning trajectories reveal differences in information weighting
}

\author{
Steffen A. Herff ${ }^{1,2,3}$, Shanshan Zhen ${ }^{4}$, Rongjun $\mathrm{Yu}^{4,5}$, Kat R. Agres ${ }^{3,6}$
}

${ }^{1}$ Digital and Cognitive Musicology Lab, École Polytechnique fédérale de Lausanne, Switzerland

${ }^{2}$ Music Cognition and Action group, The MARCS Institute for Brain, Behaviour and

Development, Western Sydney University, Australia

${ }^{3}$ Institute of High Performance Computing, Agency for Science, Technology and Research,

Singapore

${ }^{4}$ Department of Psychology, National University of Singapore, Singapore

${ }^{5}$ NUS Graduate School for Integrative Sciences and Engineering, National University of

Singapore, Singapore

${ }^{6}$ Yong Siew Toh Conservatory of Music, National University of Singapore,

Singapore

\section{Author Notes}

Correspondence concerning this article should be addressed to Steffen A. Herff, INN.

115, 1015 Lausanne, Switzerland, E-mail: steffen.herff[at]epfl.ch.

We thank Lauren Fairley, Jon Prince, and Estefanía Cano for constructive comments on an earlier draft, and Arihant Singhai, Ren Jie Tay, and Jing Wen Chai for their support during data collection. We thank Feng Lei for advice on the choice of cognitive assessment tests and 
organising training on administering the tests. The study was supported by the Singapore Ministry of Education (MOE2016-T2-1-015) awarded to Prof Yu.

Steffen A. Herff developed the paradigm, and designed, coded, as well as prepared the experiment. Kat R. Agres helped develop the experimental design and paradigm. Data collection was performed or supervised by Steffen A. Herff and Shanshan Zhen. Data was analyzed and interpreted by Steffen A. Herff. The manuscript was written by Steffen A. Herff with Shanshan Zhen, Rongjun Yu, and Kat R. Agres providing comments. The project and collaboration were initiated by Kat R. Agres, and Rongjun Yu provided lab space and equipment. All authors approved the final version of this manuscript. We have no known conflict of interest to disclose. We archived a preprint of the present work which can be accessed through: https://psyarxiv.com/kuy6p. 


\begin{abstract}
Statistical learning (SL) is the ability to generate predictions based on probabilistic dependencies in the environment, an ability that is present throughout life. The effect of aging on SL is still unclear. Here, we explore statistical learning in healthy adults (40 younger and 40 older). The novel paradigm tracks learning trajectories and shows age-related differences in overall performance, yet similarities in learning rates. Bayesian models reveal further differences between younger and older adults in dealing with uncertainty in this probabilistic SL task. We test computational models of three different learning strategies: (1) Win-Stay, Lose-Shift, (2) Delta Rule Learning, (3) Information Weights to explore whether they capture age-related differences in performance and learning in the present task. A likely candidate mechanism emerges in the form of age-dependent differences in information weights, in which young adults more readily change their behavior, but also show disproportionally strong reactions towards erroneous predictions. With lower but more balanced information weights, older adults show slower behavioral adaptation but eventually arrive at more stable and accurate representations of the underlying transitional probability matrix.
\end{abstract}

Keywords: Statistical Learning; Cognitive Assessment; Continuous Paradigm; Agerelated differences; Information Weights 


\section{Age-dependent statistical learning trajectories reveal differences in information weighting}

Statistical learning (SL) describes the ability to generate predictions based on

probabilistic dependencies in the environment. The majority of SL research focuses on early childhood development or young adults (see Krogh, Vlach, \& Johnson, 2013; Daltrozzo \& Conway, 2014 and Saffran \& Kirkham, 2018 for reviews). This approach makes intuitive sense, as SL is already present in infancy (Roseberry, Richie, Hirsh-Pasek, Golinkoff, \& Shipley, 2011; Saffran, Aslin, \& Newport, 1996). SL in older adults, however, has received far less scientific attention. Considering the worldwide increase in life expectancy and age of retirement (WHO, $2015,2017)$, it is important to further our understanding of learning in older adults. SL can be considered the outcome of a mechanism that extracts probabilistic information. Despite the overwhelming evidence for SL in humans, the fundamental mechanisms or learning strategies that allow humans to extract such probabilistic information are not yet fully understood (Krogh et al., 2013; Saffran \& Kirkham, 2018). Here, we investigate age-related differences in SL ability and mechanisms in older and younger adults. The main contribution of the present study is showing age-related differences in SL and identifying a candidate learning mechanism of SL that can capture the age-related differences.

\section{SL in older adults}

There is a growing body of evidence suggesting that older adults employ different strategies for learning, response selection, and decision-making, compared to younger adults (Hinault, Lemaire, \& Touron, 2017; Löckenhoff \& Carstensen, 2007; Mata, von Helversen, \& Rieskamp, 2010; Nassar et al., 2016; Schirda, Valentine, Aldao, \& Prakash, 2016). For example, younger participants seem more proficient in combining multiple mnemonic strategies compared to older participants (Hinault et al., 2017). Furthermore, compared to younger adults, older 
participants seem to treat positive information preferentially compared to negative information such as when recalling information about physicians or health plans (Löckenhoff \& Carstensen, 2007). Older adults also appear to utilize uncertainty of information to a lesser extent than younger participants, and younger adults show large behavioral adjustments to relatively minor prediction errors (Nassar et al., 2016). Taken together, this body of literature suggests that older adults utilize different strategies to extract information to make decisions or predictions compared to young adults. SL tasks lend themselves to investigate these differences as they test behavioral outcomes that are the result of extracting statistical regularities from the environment.

Prior research on age-related differences in SL has yielded conflicting evidence depending on the precise paradigms (e.g., deterministic vs. probabilistic) and measures (e.g., reaction times vs. accuracy) deployed. This combined with a general uncertainty about the mechanisms behind SL leaves questions about age-related shifts in information extraction strategies largely unanswered. SL of deterministic sequences (e.g., 'B' always follows 'A') is remarkably similar across age. Cherry and Stadler (1995) presented participants with four circles on a screen that flashed in a deterministic sequence, and participants predicted the next lit circle via speeded button-press. Both younger and older adults performed at ceiling in terms of accuracy, and both age groups improved reaction times with each sequence repetition. Although older adults showed overall slower reaction times, learning rates were comparable across the two groups. Other studies also reported little evidence of age-related differences in learning of deterministic sequences (Daltrozzo \& Conway, 2014; Frensch \& Miner, 1994; D. V. Howard \& Howard, 1989, 1992; Salthouse, McGuthry, \& Hambrick, 1999). However, age-related differences emerge when sequences are probabilistic - that is, governed by an underlying transitional probability (TP) matrix (e.g., ' $\mathrm{B}$ ' is most likely to follow ' $\mathrm{A}$ ' and ' $\mathrm{C}$ ' is less likely to 
follow 'A'). Curran (1997) also presented four circles to participants; however, the sequences of flashing circles switched back and forth between predetermined sequences and random sequences. The result is a TP matrix whereby each circle has a given probability to be followed by another circle. Importantly, this probability is not $100 \%$, based on the precise sequences used, as well as the interspliced random sequences. Reaction time measures revealed reduced learning in older adults compared to younger adults. Studies that provide support for better SL in younger adults on probabilistic SL tasks tend to utilize tasks where reaction time is the primary measure of performance (Curran, 1997; Feeney, Howard, \& Howard, 2002; D. V. Howard et al., 2004; J. H. Howard \& Howard, 1997). This observation is important because differences in reaction time do not necessarily reflect learning performance (Aizenstein et al., 2006). Indeed, reaction time effects may be the result of a strategic change across age in terms of a speed-accuracy trade-off (Forstmann et al., 2011; Salthouse, 1979). As a result, paradigms that require participants to respond both quickly and accurately have limited applicability for the present goal of investigating SL ability and its mechanisms, even if they do produce age-related differences (Curran, 1997). The present study also focusses on a probabilistic task, but it is concerned with age-related differences in predictive decision-making performance, rather than the speed in which learned responses are made.

To testing accuracy rather than speed, Palmer, Hutson, and Mattys (2018) presented participants with a continuous auditory stream of an artificial language with no interruptions. Accordingly, the only way of extracting individual words was by tracking the underlying transitional probabilities between syllables. This is because transitional probabilities within words are much higher than across word boundaries. After exposure to the continuous stream, participants differentiated between words from the artificial language, non-words (that did not 
exist in the artificial language), and part-words (foil words generated by combining syllables across word boundaries). Although no age-related differences occurred in differentiating words from non-words, younger participants outperformed older participants when differentiating words from part-words (Palmer et al., 2018; Palmer \& Mattys, 2016). This result suggests that older adults may utilize different strategies than younger adults to extract statistical information. However, such differences may also be due to an age-related decline in cognitive function (e.g., memory). To account for possible differences in cognitive function, we also collect cognitive assessment data from the participants in our experiment.

To capture potential differences in learning strategies, we analyze learning trajectories between groups of younger and older adults. Rather than analyzing overall SL performance alone, we focus on individuals' learning trajectories (slopes), as previous work suggests this measure provides valuable insight into individuals' cognitive capacities and the time course of learning novel information (Kaufman et al., 2010; Misyak, Christiansen, \& Tomblin, 2010; Siegelman, Bogaerts, Christiansen, \& Frost, 2017). Learning trajectories are of particular interest for the present study because the time course of information integration may more accurately characterize age-related differences than the absolute performance. Analyzing learning trajectories can also be especially informative when the underlying TP matrix contains transitions where the most likely next event is by far the most probable one (high-certainty state), as well as transitions where the most likely next event is less obvious (low-certainty state) (Shafir, Reich, Tsur, Erev, \& Lotem, 2008). This is because a TP matrix with various different states of uncertainty allows for more precise observation of participants' information integration, which in turn allows computational models to provide a far more detailed investigation of differences in participants' learning strategies. 


\section{Statistical Learning Mechanisms}

In the present work, we consider three learning mechanisms: (1) Win-Stay, Lose-Shift, (2) Delta Rule Learning based on probability spectrum, (3) Information Weights. Investigating these three learning mechanisms and exploring whether they can explain age-related differences in SL constitutes the main focus of the present work. The choice of these three mechanisms was predominantly guided by their conceptual simplicity and presence in the literature, as well as ease of implementation.

\section{Win-Stay, Lose-Shift}

The first learning mechanism we consider captures whether participants - when forming a prediction - predominantly rely on the outcome from their last response when faced with the same decision. Such Win-Stay, Lose-Shift strategies are commonly observed in decision-making tasks (Nowak \& Sigmund, 1993; Worthy, Hawthorne, \& Otto, 2013) and previous research indicates age-related differences in decision-making in terms of Win-Stay, Lose-Shift usage. For example, older participants tended to rely more strongly on a Win-Stay, Lose-Shift mechanism in a probabilistic inference task (Mata et al., 2010). Computational modelling of Win-Stay, LoseShift shows that, in theory, it is an effective strategy for language learning (Matsen \& Nowak, 2004). Participants could theoretically utilize a Win-Stay, Lose-Shift strategy to solve a SL task. However, for any SL paradigm, predominantly relying on such a strategy would be potentially problematic. This is because SL tasks are often designed with the assumption that participants continuously sample information from the environment to extract statistical regularities, rather than only when they are prompted to respond and only from the last time they provided a response. Relying on a Win-Stay, Lose-Shift mechanism would suggest that participants deploy a simple response heuristic to achieve statistical learning without extracting the full underlying 
set of transitional probabilities. This is because Win-stay, Lose-Shift does not rely on extracting statistical properties - instead it relies exclusively on memory of the last relevant response. In the case that we observe the Win-Stay, Lose-Shift strategy in the present task, a stronger reliance on this strategy is hypothesized in older participants (Mata et al., 2010).

\section{Delta-Rule Learning based on probability spectrum}

The second mechanism we consider aims to capture whether participants responses are predominantly driven by two factors. First, the distance between the currently perceived transitional probabilities to the new estimated probabilities after receiving new information (e.g., feedback). Second, the absolute values of the estimated real probability. In other words, it measures whether participants more strongly adjust their predictions when the perceived probabilities are further away from the true transitional probabilities (e.g., $\Delta=.5$ with $P_{\text {Perceived }}=$ .25 and $P_{\text {Real }}=.75$ vs. $\Delta=.25$ with $\left.P_{\text {Perceived }}=.5, P_{\text {Real }}=.75\right)$, and whether this adjustment differs depending on whether it occurs towards the low or high end of the probability spectrum (e.g., $\Delta$ $=.2$ with $P_{\text {Perceived }}=.3, P_{\text {Real }}=.5$ vs. $\Delta=.2$ with $\left.P_{\text {Perceived }}=.55, P_{\text {Real }}=.75\right)$ ? Adjusting behavior based on the observed discrepancy between a prediction and the observed reality is an intuitive and well-established learning mechanism (Rescorla \& Wagner, 1972). This learning mechanism can be described as 'delta-rule' learning, because response probabilities change by a proportion of the prediction error (here, the difference between the predicted probability of a transition, and the real probability of a transition) (Greve, Cooper, Kaula, Anderson, \& Henson, 2017). In a probabilistic SL task, effective use of delta-rule learning relies on an estimate of one's own perception of the transitional probabilities, as well as the true underlying probabilities. Prior literature suggests that younger adults, compared to older adults, show relatively large adjustments even to small prediction errors (Nassar et al., 2016). Furthermore, the present 
implementation of delta-rule learning also allows the mechanism to vary depending on whether the true probability is likely or unlikely. This implementation decision is motivated by findings in the literature suggesting that SL may differ between age groups and as a function of task complexity (Curran, 1997; Feeney et al., 2002; D. V. Howard et al., 2004; J. H. Howard \& Howard, 1997; Palmer \& Mattys, 2016). Given that we cannot assume that learning a less likely transition (e.g., $\left.P_{\text {Real }}=.5\right)$ is equally difficult as learning a more likely transition $\left(P_{\text {Real }}=.75\right)$, we need this additional mechanism to capture potential age-related differences.

Observing delta-rule learning in the present probabilistic SL task would provide evidence that participants are extracting the underlying statistical regularities. If delta-rule learning describes participants' behavior in the present task, we predict younger adults to adjust their behavior more rapidly than older adults, based on previous findings (Nassar et al., 2016). Furthermore, if task difficulty differs as a function of transitional probability, we expect to see stronger delta-rule learning in more probable transitions. If this is the case, we also hypothesize an interaction with age, whereby older participants' delta rule learning decreases to a greater extent compared to younger adults as transitional certainty increases. This is because the increased task complexity may function as a greater obstacle to the older adults compared to the younger adults. It is worth noting that estimating the delta required for delta-rule learning can be difficult, particularly in a probabilistic task.

\section{Information Weights}

In the present work, we propose a learning mechanism that is more parsimonious than the Delta-Rule model, as it does not rely on estimating delta, and yet would be effective in extracting statistical regularities and reveal age-related differences - Information Weights. The model simply assesses the weights (change in response probabilities) that younger and older adults 
attach to positive (e.g., 'B' follows 'A') and negative (e.g., 'B' does not follow 'A') observations. Effectively, this cognitive model simplifies the mechanism behind statistical learning to a continuous sampling of information with a 'positive' weight that reflects increasing the likelihood of making a particular choice when the specific transition is observed in the sequence, and a 'negative' weight that reflects decreasing the likelihood of making the particular choice when the specific transition is not observed. As a result, with only two parameters ('positive' and 'negative' weight) that differ between individuals or groups, the model could be able to explain differences in response behavior. This mechanism can be understood as a generalization of Thorndike's law of effect (Thorndike, 1898). A model of this learning mechanism allows the comparison of participants in regards to how willing they are to update their response probabilities. It also allows an assessment of whether participants rely more on positive or negative information. This is an interesting perspective that the previous delta-rule model cannot capture, but may be an important consideration in the context of age-related differences in SL. Prior research suggests general age-related differences in the processing of positive (prediction was fulfilled) and negative (prediction was not fulfilled) feedback, with older participants showing a tendency to rely more on positive feedback compared to younger adults (Eppinger \& Kray, 2011; Ferdinand \& Kray, 2013). These studies provide explicit feedback, however, and do not take place within the framework of SL. If participants' response patterns can be modelled through Information Weights and not through Win-Stay, Lose-Shift, then this would suggest that participants are extracting the underlying statistical regularities. We predict younger adults to show higher information weights than older adults. Furthermore, based on the literature reviewed above, we predict older adults to have a larger positive-to-negative weight ratio when compared to younger adults. 


\section{SL, Cognitive Function, and the present paradigm}

An additional consideration when looking at SL differences in older adults is the possibility that poorer performance may reflect an age-related decline in cognitive function (e.g., memory). A decline in cognitive function could lead to lower performance due to task-specific requirements (e.g., auditory memory) or because of a direct influence of cognitive function on SL. Much effort has been made to investigate the relationship between SL and cognitive function (Feldman, Kerr, and Streissguth, 1995; Kaufman et al. 2010; Siegelman, Bogaerts, and Frost, 2017). However, despite SL's crucial involvement across sensory modalities (Creel, Newport, \& Aslin, 2004; Kirkham, Slemmer, \& Johnson, 2002) (Moldwin, Schwartz, \& Sussman, 2017) research attempting to link SL to traditional cognitive assessments has yielded limited evidence for a direct link between SL and cognitive function (e.g., $r$ from -.06 to.19 in Feldman, Kerr, and Streissguth, 1995; Kaufman et al. 2010; Siegelman, Bogaerts, and Frost, 2017). In addition, previous attempts to use SL as a measure of individual aptitude or to link it to various established measures of cognitive function have been plagued by a plethora of difficulties (Siegelman, Bogaerts, \& Frost, 2017). These include low test-retest reliability ( $r=.44$ in Kaufman et al., 2010), and low performance in the participants $(21-47 \%$ of participants at chance level, see Siegelman, Bogaerts, and Frost, 2017 for a review). Consequently, the low correlations with measures of cognitive function could either be a product of the aforementioned methodological issues or indeed indicative that SL is mostly independent of other cognitive skills. To capture cognitive function as a possible covariate and test its contribution to the question of whether SL is directly influenced by cognitive function, we administer a battery of cognitive assessments, further described in the method section.

\section{The Present Paradigm}


Based on Siegelman, Bogaerts, and Frost's (2017) criticism of existing SL paradigms, a new auditory SL paradigm that focuses on learning trajectories was developed (Herff, Nur, Lee, Lee, \& Agres, 2019). In this task, participants listened to a continuous stream of four different sounds and were occasionally prompted to indicate the most likely next sound. The paradigm showed high test-retest reliability in older adults $(r=.84)$, and correlated well with measures of cognitive function $(r=.56)$. Furthermore, the task satisfies the needs outlined in the previous section: It is probabilistic, measures accuracy, tracks learning trajectories, and the TP matrix can be adjusted to contain low- and high-certainty transitions. The auditory domain is also a promising target to measure SL ability and link it to cognitive ability. This is because the auditory domain specialize in processing stimuli that unfold in time (Pérez-González \& Malmierca, 2014) and relies heavily on extracting statistical information from the environment (Agres, Abdallah, \& Pearce, 2018; Barascud, Pearce, Griffiths, Friston, \& Chait, 2016; Sohoglu \& Chait, 2016). However, similar to previous SL paradigms, many participants performed at chance level, and a relatively small sample size was used $(n=27)$ (Herff, Nur, et al., 2019). The authors suggested deploying more trials and modifying the task to be multi-modal.

Consequently, we use Herff, Nur, et al. (2019)'s SL paradigm to capture learning trajectories, incorporating more trials (150 instead of 50), a multi-modal implementation (auditory-visual), and a new TP matrix that accommodates low- and high-certainty states. Further details of the paradigm are described in the method section.

\section{Aim and Motivation}

In summary, the present study investigates age-dependent differences in SL. We utilize a continuous, multi-modal, probabilistic paradigm to reveal SL trajectories in younger and older 
adults. The probabilistic TP matrix governing the task contains low- and high-certainty transitions to help us identify potential learning strategies that capture SL and potential agerelated differences. Predominantly, we explore whether three mechanisms of learning can describe SL and potential age-related differences, specifically: (1) Win-Stay, Lose-Shift, (2) Delta Rule Learning based on probability spectrum, and (3) Information Weights. To account for the potentially moderating effect of age-related differences in cognitive function, we also explore whether traditional cognitive assessments correlate with SL performance in this task and can explain response differences between the age groups.

\section{Method}

\section{General Procedure}

After providing informed consent, participants took part in a cognitive assessment ( 30min), followed by the SL paradigm ( $\sim 45 \mathrm{~min})$. The present data collection was part of a large EEG project collaboration between the Agency for Science, Technology and Research (A*STAR) and the National University of Singapore (NUS). Analysis of the collected EEG data will be reported elsewhere.

\section{Participants}

Data from 40 younger adults were recorded from the student population at the National University of Singapore $\left(M_{\text {age }}=21.4 S D_{\text {age }}=2.7\right) ; 40$ older adults (defined as $60+$ years old) were recruited from the community $\left(M_{a g e}=66.7, S D_{a g e}=4.2\right)$. Participants were required to have normal or corrected-to-normal hearing, be literate in English, be able to provide informed consent, and be able to travel to the study site independently. Participation was reimbursed with SGD 40. The study was IRB approved (S-17-372). 


\section{Stimuli and Equipment}

\section{Statistical Learning Paradigm}

The present study deployed a continuous SL paradigm designed to capture learning trajectories (Herff, Nur, et al., 2019). A long series of states was generated whereby each state could be one of four options. The four states were differentiated through sound (sine waves at $165 \mathrm{~Hz}$ (E3), $220 \mathrm{~Hz}$ (A3), $294 \mathrm{~Hz}$ (D4), $392 \mathrm{~Hz}$ (G4), each 500ms in duration). Participants heard this long series of four possible states, and the series paused every 7.5 to $11.5 \mathrm{sec}$ (15 - 23 tones), at which point the participants were prompted to indicate which tone they thought would occur next. The number of tones between stopping points was variable to avoid potential expectancy effects of when the next interruption would occur. After a response, the sequence would continue. The sequence was instantiated in both the auditory and visual modality. Four horizontally aligned circles on the screen were associated with the four sounds (in order of lowest to highest pitch, left to right). For each tone, a circle flashed as the respective sound was played. After each stop in the sequence, participants indicated their response by clicking on the circle that they thought would occur next (four alternative forced-choice). The response window was not timed. Participants did not receive explicit feedback, however, since the sequence continued after each response, feedback was implicitly provided by the following state and whether or not it matched the participants' prediction. In total, 150 responses (trials) per participant were collected.

\section{Transitional Probability Matrix}

The TP matrix governing the four states can be seen in Figure 1. The overall probability of each state is identical (25\%). Two states ('A',' D', purple in Figure 1) are considered highcertainty states, as the most likely next state is evident with a $75 \%$ transitional probability. The 
other two states ('B', 'C', blue in Figure 1) are low-certainty states, as the most likely next state is less evident with only a $50 \%$ transitional probability. For example, the most likely state after A is $\mathrm{B}$, with a probability of $75 \%$. The most likely state after ' $\mathrm{B}$ ' is ' $\mathrm{D}$ ', with a probability of only $50 \%$. The probability of repeating a state is zero, thus a response indicating repetition is considered a rule violation. Cumulative Rule Violations (CRV) as well as Cumulative High Probability Pathway Choices (CHPC) are the two measurements of SL performance used here. CRV refers to the number of rule violations (responses indicating a repetition; red arrows in Figure 1) accumulated up to a given trial. CHPC refers to the number of high-probability responses (responses correctly identifying the most likely next state; green arrows in Figure 1) accumulated up to a given trial. Thus, good performance is indicated by high CHPC and low CRV (Herff, Nur, et al., 2019). Note, that whilst likely correlated, the two measures assess different aspects of SL, as CHPC captures whether participants learn the most likely next event, and CRV assesses whether participants learn to exclude impossible outcomes. In other words, on a given trial, participants may avoid a rule violation but still not pick the high-probability choice - there are two other low-probability choices (black arrows in Figure 1). Similarly, participants may not make the correct high probability choice, but that does not necessarily mean that they chose a rule violation (repetition).

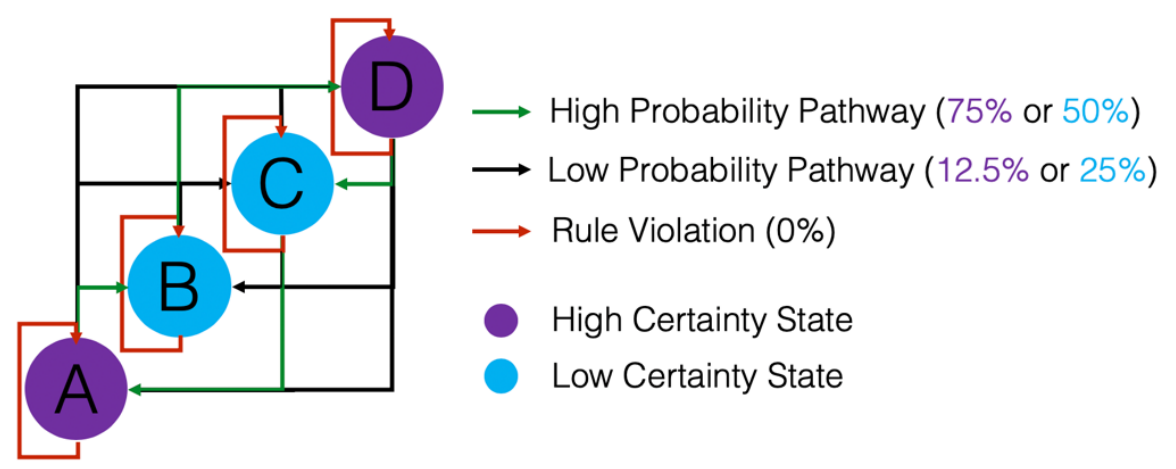


Figure 1: Schematic representation of the TP matrix. The two main measures of SL performed used here are Cumulative Rule Violations (CRV, accumulation of response associated with a red arrow) and Cumulative High Probability Choices (CHPC, accumulation of responses associated with a green arrow). Because the most likely next state is clearer (75\%, purple) in state 'A' and ' $\mathrm{D}$ ' compared to states ' $\mathrm{B}$ ' and 'C' (50\%, blue), states A and D are considered high-certainty states, and states B and C are considered low-certainty states.

\section{Cognitive Assessment}

A battery of cognitive tests was administered to prevent any confounding of group differences in general cognitive ability with SL learning trajectories. Furthermore, prior research has shown conflicting evidence as to whether SL is predicted by cognitive ability (Feldman et al., 1995; Herff, Nur, et al., 2019; Kaufman et al., 2010; Siegelman, Bogaerts, \& Frost, 2017). Though not the main focus of this study, we hope that collecting cognitive ability data in addition to the present SL paradigm may also contribute to the debate. The selection of cognitive tests was informed by consulting a clinician specialized in working with auditory learning tasks in an older adult population (see Feng et al., 2017; Tan et al., 2018 for studies utilizing the same battery of tests). The tests aim to provide an overview of cognitive ability that may be relevant to auditory learning tasks in general.

Specifically, the battery of cognitive tests deployed here comprises the Rey Auditory Verbal Learning Test (RAVLT) (Rey, 1958), Digit Span task (backwards and forwards), Verbal Fluency task (see Randolph, Braun, Goldberg, \& Chase 1993), Symbol Digit Modality Test (Smith, 1982) in written (DSW) and verbal (DSV) form, and Color Trails Test (D'Elia, Satz, Uchiyama, \& White, 1996). All assessors were formally trained and the tests were administered as described in the Neuropsychological Assessments Training Manual for Assessors (Yu, 2018). A short summary of each test follows below. 
RAVLT. The test comprises multiple parts. In part one, participants listen to a list of 15 words (List-A) and then attempts to recall them. This procedure is repeated five times, and the number of correct recalls is counted after each iteration. In the models, this is coded as RAVLTI to RAVLT5. In the second part, the participant listens to a different 15-item word list (List-B), and the number of correctly recalled items is coded as RAVLTB. Afterwards, participants are asked to recall the items from List-A again, and the number of correctly recalled items is coded as RAVLTRECA. After a delay, filled with the Digit Span Test and Color Trail test (see below), the RAVLT assesses delayed recall by requiring participants to recall the items of List-A once more. The number of correctly recalled items is coded as RAVLTDelayedRacall. In the third part of the RAVLT, participants listen to a list of 50 items, 15 of which were in List-B, and the participants aim to identify words that have been presented before. The number of correctly recognised words is coded in the models as RAVLTRecognition. This RAVLT assesses verbal memory in terms of recognition as well as recall.

Digit Span Task. This task consists of two parts. In the first part, participants are asked to listen to short sequences of numbers and repeat them verbally. The task consists of two items for each sequence length. If both sequences are not correctly repeated, the task stops and the total number of correctly recalled strings is coded as DigitSpanFWD. Afterwards, the same task is repeated with different numbers. This time, however, participants are required to repeat the numbers backwards. The number of correctly recalled sequences is coded as DigitSpanBWD. The Digit Span tasks assess working memory capacity.

Color Trails Test. The test consists of two parts. In part one, participants connect numbered circles in ascending order on a sheet of paper. In the second part, participants connect numbers and letters, by alternating between numbers (in ascending order) and letters (in 
alphabetic order). The test assesses visual attention and task switching capability. Time to completion is measured separately for the two parts, and both are included in the models, referred to as ColorTrail1 and ColorTrail2.

Verbal Fluency Task. This task requires participants to name as many animals as possible in 60 seconds. The number of different animal names is coded as SemanticFluencyAnimals in the models. The test assesses linguistic storage and retrieval.

Symbol Digit Modality Test. In the first part, participants are provided with a visual key that links the numbers ' 1 to 9' to nine different visual symbols. Participants then have 90 seconds to transcribe a list of symbols as their matching number. The number of correctly linked symbols is coded as DigitSymbolWritten. In the second part, participants are provided with a new response sheet and repeat the task; however, this time they speak the number aloud, rather than writing it on the sheet. The number of correctly linked symbols in the second part is coded as DigitSymbolVerbal. The tests assess association memory, divided attention, and visual scanning.

\section{Results}

The results are structured in three parts. First, we report overall SL performance in both age groups and how they differ between low- and high-certainty states. Then, we attempt to model the results through three learning mechanisms: (1) Win-Stay, Lose-Shift; (2) Delta Rule Learning based on probability spectrum; (3) Information Weights. Finally, we explore the relationship between the battery of cognitive assessments and SL performance.

\section{Statistical Learning, Age, and Certainty}

A total of 12,000 responses were collected, evenly distributed across the four states $(\mathrm{A}=$ $25.57 \%, \mathrm{~B}=25.92 \%, \mathrm{C}=24.02 \%, \mathrm{D}=24.48 \%$ ). We used a simulation-based approach to assess 
chance and ideal performance (see Supplement S0). 95\% CIs were calculated around simulated guessing participants and simulated ideal Bayesian learners. The results are summarized in Table 1, and Figure 2 depicts overall learning trajectories.

Table 1. SL Performance Summary

Note. More than chance CHPC, and less than chance CRV indicate successful learning of the TP matrix.
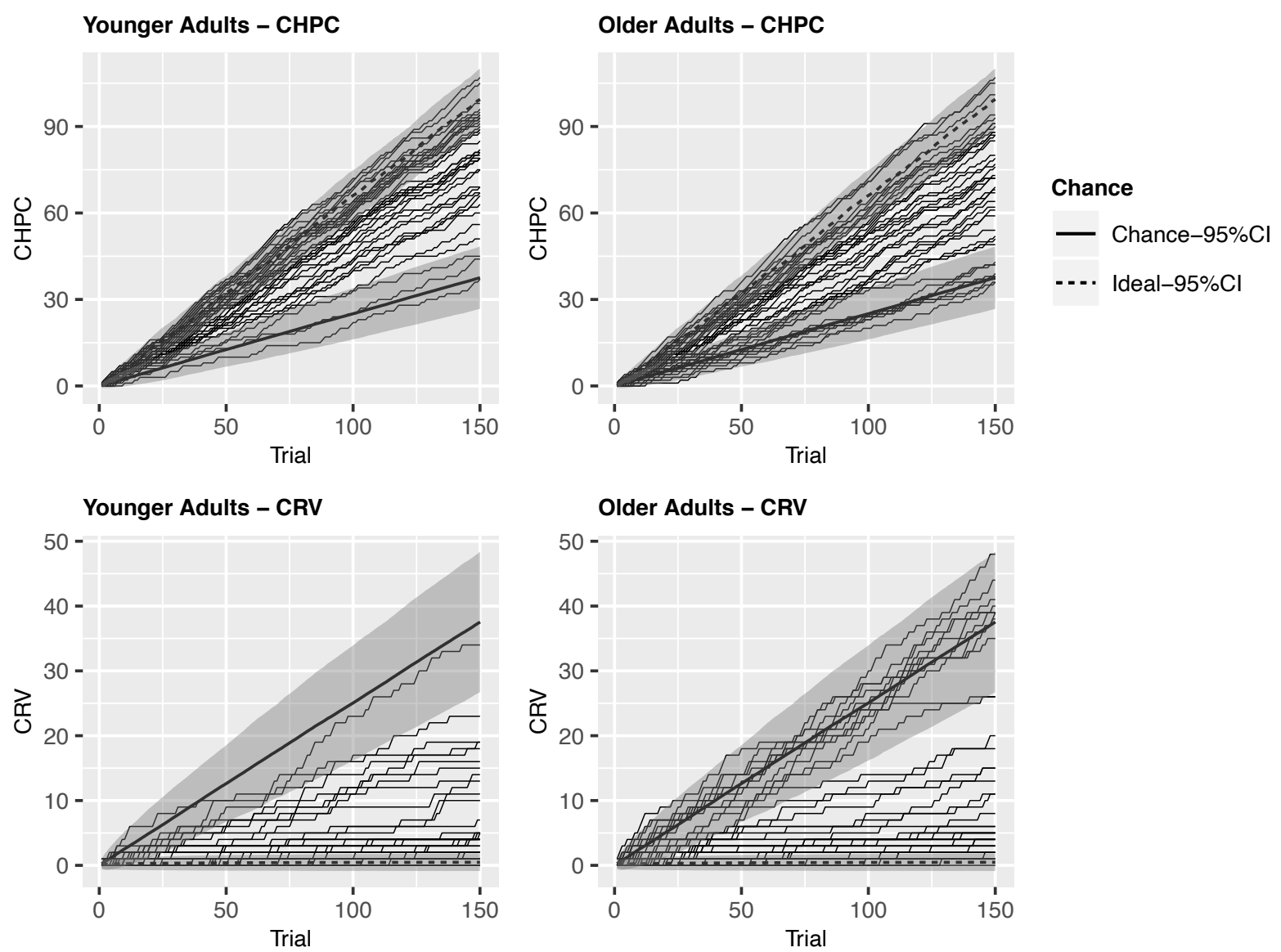
Figure 2: Overall performance in the SL task. The top row shows cumulative high-probability choices (CHPC). The bottom row shows cumulative rule violations (CRV). The left column shows data from younger adults, and the right column shows data from older adults. Each thin line represents one participant. The bold solid lines represent chance performance. Above chance in CHPC, and below chance in CRV, indicates good performance. The dotted line shows a theoretical ideal performer. The grey bands represent $95 \%$ CIs around chance and ideal performance.

A generalized Bayesian mixed-effects model predicted the responses that lie on the highprobability pathway. The model was provided with a fixed effect for Trial (1-150, representing the learning trajectory over the course of the experiment), Age (younger adults vs older adults), Certainty (low-certainty state vs. high-certainty state), as well as all interactions. The model was also provided with random effects for Participant and the precise Sequence presented. Further information about the models can be found in Supplement S1. We report coefficient estimates $(\beta)$, estimated error $(E E)$ in the coefficients, as well as evidence $(O d d s)$ ratios for the individual hypotheses (a given coefficient being larger or smaller than zero). For convenience, we denote effects with "*” as those which can be considered 'significant' at an $\alpha=.05$ level. This corresponds to odds ratios $>=19$ (odds 95/5 = 19) (Milne \& Herff, 2020).

$\operatorname{Trial}\left(\beta=.14, E E_{\beta}=.05, \operatorname{Odds}(\beta>0)=579.65^{*}\right)$ predicted the probability of highprobability pathway responses, indicating that learning took place. Age $\left(\beta=-.31, E E_{\beta}=.09\right.$, $\operatorname{Odds}(\beta<0)>9999 *)$ also carried predictive value, with younger adults overall being more likely to produce high-probability pathway responses. The low-certainty states led to overall fewer high-probability pathway responses $\left(\beta=-.61, E E_{\beta}=.07, \operatorname{Odds}(\beta<0)>9999 *\right)$, indicating that participants were able to discern the differences between states in the TPs. The LowCertainty $x$ Trial interaction $\left(\beta=-.31, E E_{\beta}=.07, O d d s(\beta<0)>9999 *\right)$ predicted reduced 
high-probability pathway responses in low-certainty states as the experiment progresses. This can be seen in Figure 3 in the positive slope for the high-certainty states, and the negative slope for the low-certainty states. The Trial $x$ Certainty $x$ Age interaction $\left(\beta=.15, E E_{\beta}=.05, \operatorname{Odds}(\beta>\right.$ $0)=733.69^{*}$ ) showed that (as the experiment progresses) younger adults' likelihood to produce high-probability pathway responses decreases more strongly in the low-certainty states compared to older adults. Figure 3 depicts this finding - the blue line (low-certainty state) has a steeper slope for younger adults (left panel) than older adults. Importantly, the Trial x Age interaction did not carry predictive value $\left(\beta=-.03, E E_{\beta}=.03, \operatorname{Odds}(\beta<0)=3.88\right)$. This means that learning trajectories in high-certainty states were comparable between the two age groups, as shown in Figure 3 (the red lines, depicting high-certainty states, have similar slopes across age groups).

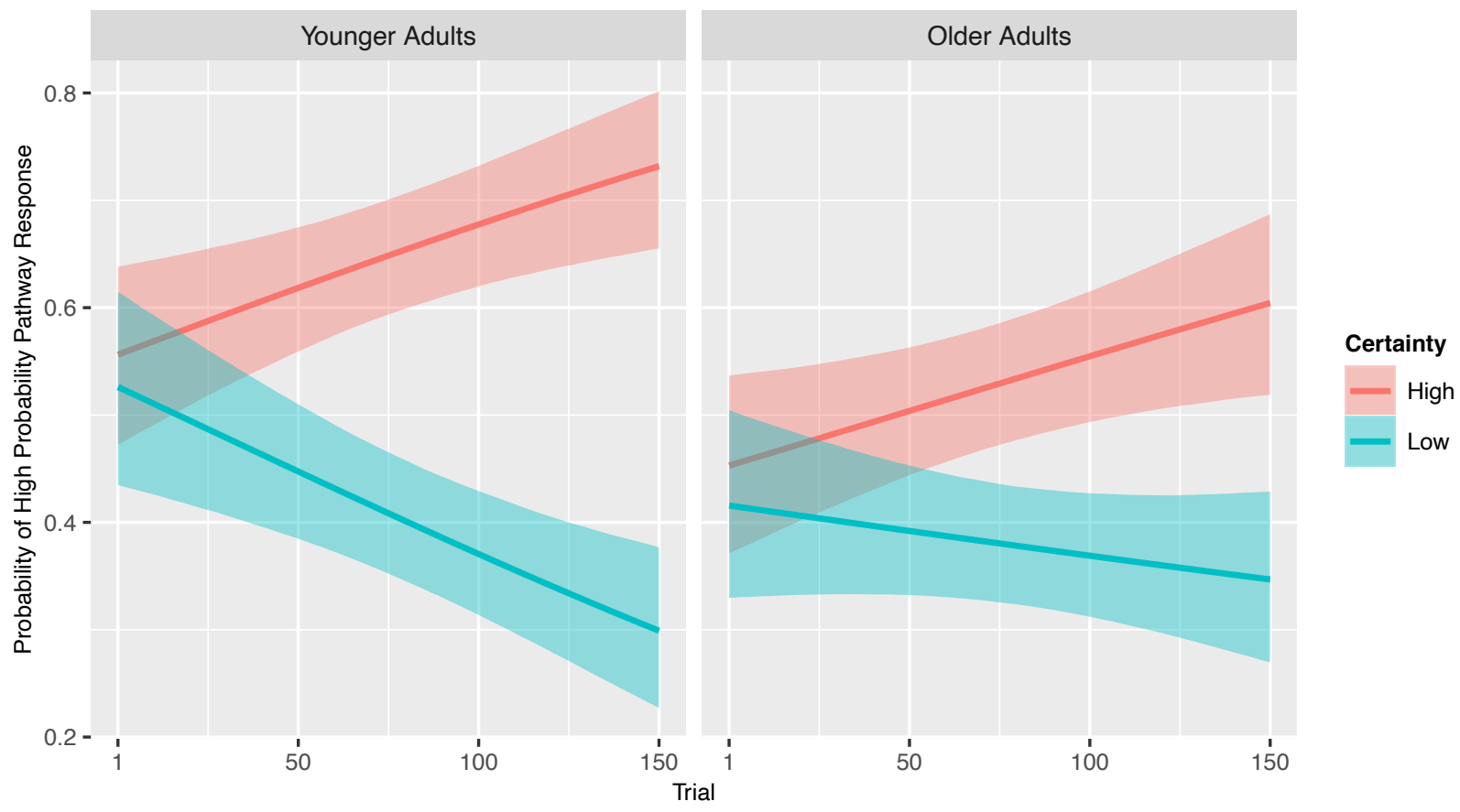

Figure 3. Effects of age and certainty state on SL as measured by the probability of producing a response compatible with the high-probability pathway. Both age groups show clear learning trajectories. Younger adults 
show a higher intercept at the beginning of the experiment compared to older participants. Learning trajectories (slopes) are comparable between the two age groups on high-certainty states (red lines). Interestingly, both groups appear to underestimate the probability of the most likely response in the low-certainty states (blue lines). This is particularly pronounced in the younger adults, who, for low-certainty states, produced increasingly fewer responses over the course of the experiment that lie on the high-probability pathway. The bands indicate $95 \%$ CIs.

For CRVs, we combined the data from low- and high-certainty states, as both have 0\% TPs of repeating states. Age $\left(\beta=.34, E E_{\beta}=.14, \operatorname{Odd} s(\beta>0)=136.40^{*}\right)$ predicted the probability of rule violations, with older adults $(M=0.0972, S D=0.2963)$ on average showing more rule violations than younger adults $(M=0.0463, S D=0.2102)$. Both $\operatorname{Trial}\left(\beta=-.03, E E_{\beta}=\right.$ $.03 \operatorname{Odds}(\beta>0)=5.61)$ as well as the Trial $*$ Age interaction $\left(\beta=.1, E E_{\beta}=.04, \operatorname{Odds}(\beta<0)=\right.$ 1.60) did not show an effect. This is most likely because of the small number of rule violations (see Supplement S1 for a summary and the risk ratios of the SL, age, and certainty models). Due to the overall smaller degree of variability in the CRV data, the following models focus on CHPC.

\section{Statistical Learning Mechanisms}

To further explore the cognitive basis of age-related differences in SL, we tested three cognitive models. In particular, we hoped to reveal a mechanism that captures the age-related differences in SL of low- and high-certainty states (see Figure 3).

\section{Model 1: Win-Stay, Lose-Shift Strategy}

The first model assessed whether participants predominantly used the outcome from their previous response to the same state when forming a prediction. We did not find evidence for the 
LastPredHPP x LastPredCorrect interaction, suggesting participants did not predominantly rely on the information of their last prediction $\left(\beta=-.05, E E_{\beta}=.15\right.$, $\left.\operatorname{Odds}(\beta<0)=1.80\right)$. Low evidence for the LastPredHPP $x$ LastPredCorrect $x$ OlderAdult interaction shows that this behavior did also not differ between age groups $\left(\beta=-.25, E E_{\beta}=.20, \operatorname{Odds}(\beta<0)=8.88\right)$, and therefore does not explain the age-dependent behavior towards low-certainty states (see Supplement S3.1 for the full model).

\section{Model 2: Delta Rule learning based on probability spectrum}

Both age groups deployed a learning mechanism whereby they adjusted their behavior more strongly for larger errors as captured by strong evidence for the ActualMinusResponseProbs coefficient $\left(\beta=2.20, E E_{\beta}=.18, \operatorname{Odds}(\beta>0)>9999^{*}\right)$. The ActualMinusResponseProbs $x$ OlderAdult interaction term reveals that this adjustment was larger in the young adults than the older adults $\left(\beta=-.71, E E_{\beta}=.24, O d d s(\beta>0)=733.69 *\right)$. Evidence for the ActualMinusResponseProbs x StateSpecificReponseProbs interaction shows that both groups also adjusted their behavior depending on where in the probability spectrum the incongruence between believed and real probability occurs, with stronger behavioral changes towards the higher end $\left(\beta=.43, E E_{\beta}=.24, \operatorname{Odd}(\beta>0)=25.47^{*}\right)$. However, we found no evidence that this incongruency mechanism differs between the age groups in the ActualMinusResponseProbs $x$ StateSpecificReponseProbs $x$ OlderAdult interaction term ( $\beta=$ $\left..34, E E_{\beta}=.32, \operatorname{Odds}(\beta<0)=5.84\right)$. As a result, this model does not explain the age-dependent differences in low-certainty responses shown in Figure 3 either (see Supplement S3.2 for the full model).

\section{Model 3: Information Weights}


The third model is a parsimonious explanation and simply assesses the weights that younger and older adults attach to positive (e.g., B follows A) and negative (e.g., B does not follow A) observations. Because the Bayesian models provide slope coefficients of behavioral change in both age groups at two different transitional probabilities for the high-probability pathway, we have two equations for each age group, each with two unknowns. As a result, we can use Gaussian elimination (see Supplement S3.3) to obtain the weights of older adults (PositiveWeight $_{\text {olderAdult }}=.27$, NegativeWeight $\left.t_{\text {olderAdult }}=-.37\right)_{\text {and younger adults }}$ (PositiveWeight $_{\text {YoungerAdults }}=45$, NegativeWeight $\left.{ }_{\text {YoungerAdult }}=-.79\right)_{\text {attached to the continued }}$ sampling of positive and negative observations in a simplified decision-making model. The resulting weights are seen in Figure 4.

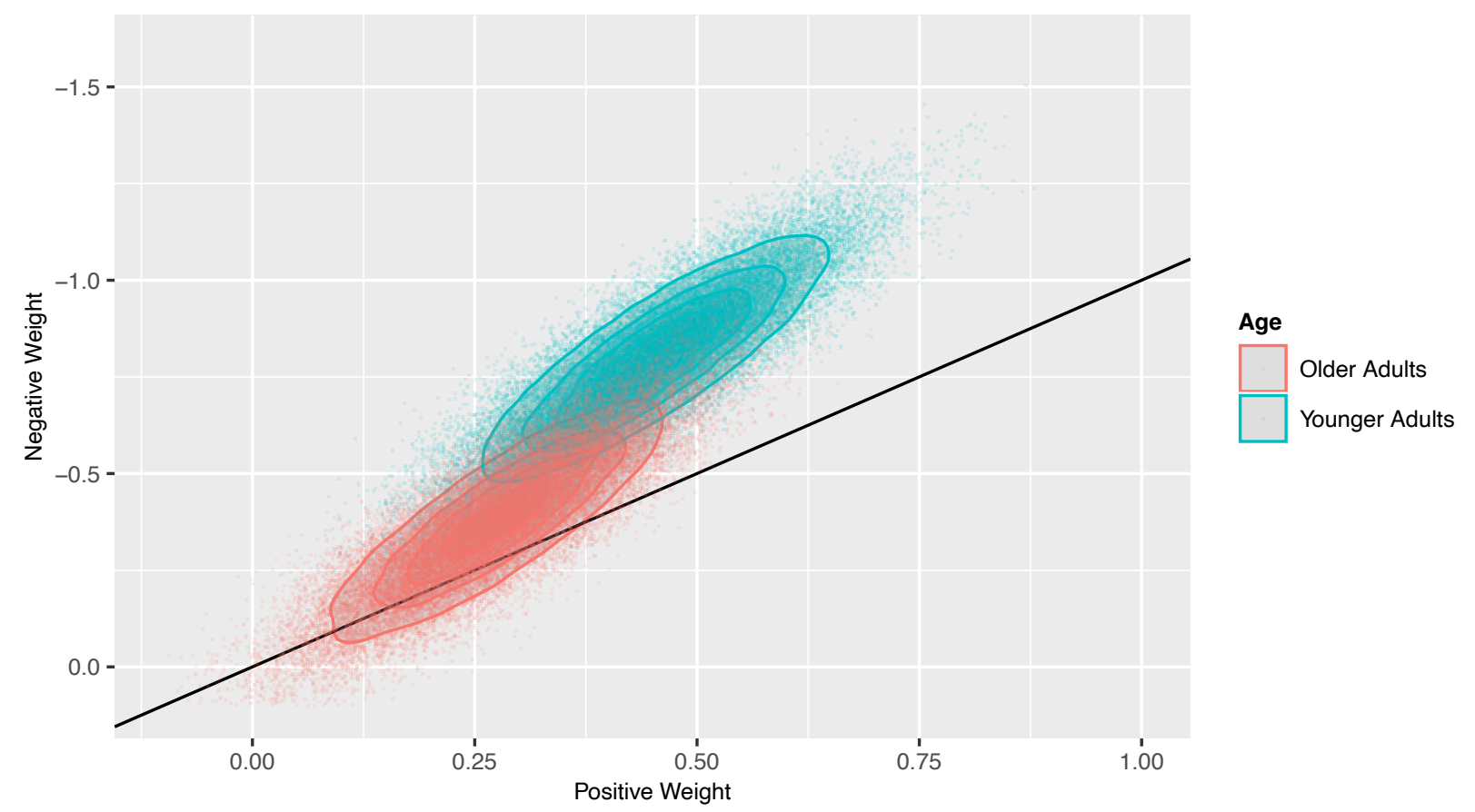

Figure 4. Estimated weights distribution to positive and negative observations in both age groups. Positive weights indicate predicted change towards providing a given answer, after observing a transition suggesting this answer (the positive number indicates that the probability increases). Negative weights indicate predicted change away from 
providing a given answer, after observing a transition which suggests that this is not the answer (the negative number indicates that the probability decreases). Both groups show clear signs of learning by using both positive and negative observations. This is indicated by the non-zero weights on both axes for both groups, and by the fact that in both groups, positive weights all fall within the range of positive numbers (increase in probability to provide the response), and negative weights all fall within the range of negative numbers (decrease in probability to provide the response). Younger adults show larger sways in their predictions as shown by the larger weights on both axes compared to older adults. Although both younger and older adults weight negative observations more strongly than positive, this is substantially more pronounced in the younger adults group.

To obtain the distribution of weights in Figure 4, the information weights for both groups were calculated after each iteration of the Bayesian Model. Since the model ran on 10,000 iterations, with 1000 warmups on four cores, Figure 4 uses the data of a total of 36,000 posterior distributions. A Hotelling $\mathrm{T}^{2}$ test using 10,000 permutations shows a significant difference between the distribution of information weights in older adults from that of younger adults $\left(t^{2}(2,71997)=112447.7, p=<.0001\right)$. Further support was found by calculating KullbackLeibler divergence on the probability density functions of younger and older adults' information weights. The divergence across age group $\left(D_{K L}\left(\mathrm{PDF}_{\text {OlderAdults }} \| \mathrm{PDF}_{\text {YoungerAdults }}\right)=3.1054\right)$ is substantially larger compared to the Kullback-Leibler divergence distribution obtained from 10,000 random permutations of the Age group vector $\left(D_{K L-M e a n}\left(\mathrm{PDF}_{\mathrm{GroupA}} \| \mathrm{PDF}_{\mathrm{GroupB}}\right)=.00012\right.$, $\left.D_{K L-S D}\left(\mathrm{PDF}_{\mathrm{GroupA}} \| \mathrm{PDF}_{\mathrm{GroupB}}\right)=.00008\right)$. In summary, we found strong support that the younger and older adult cohorts operate on different information weights. This can also be seen in Figure 5. 


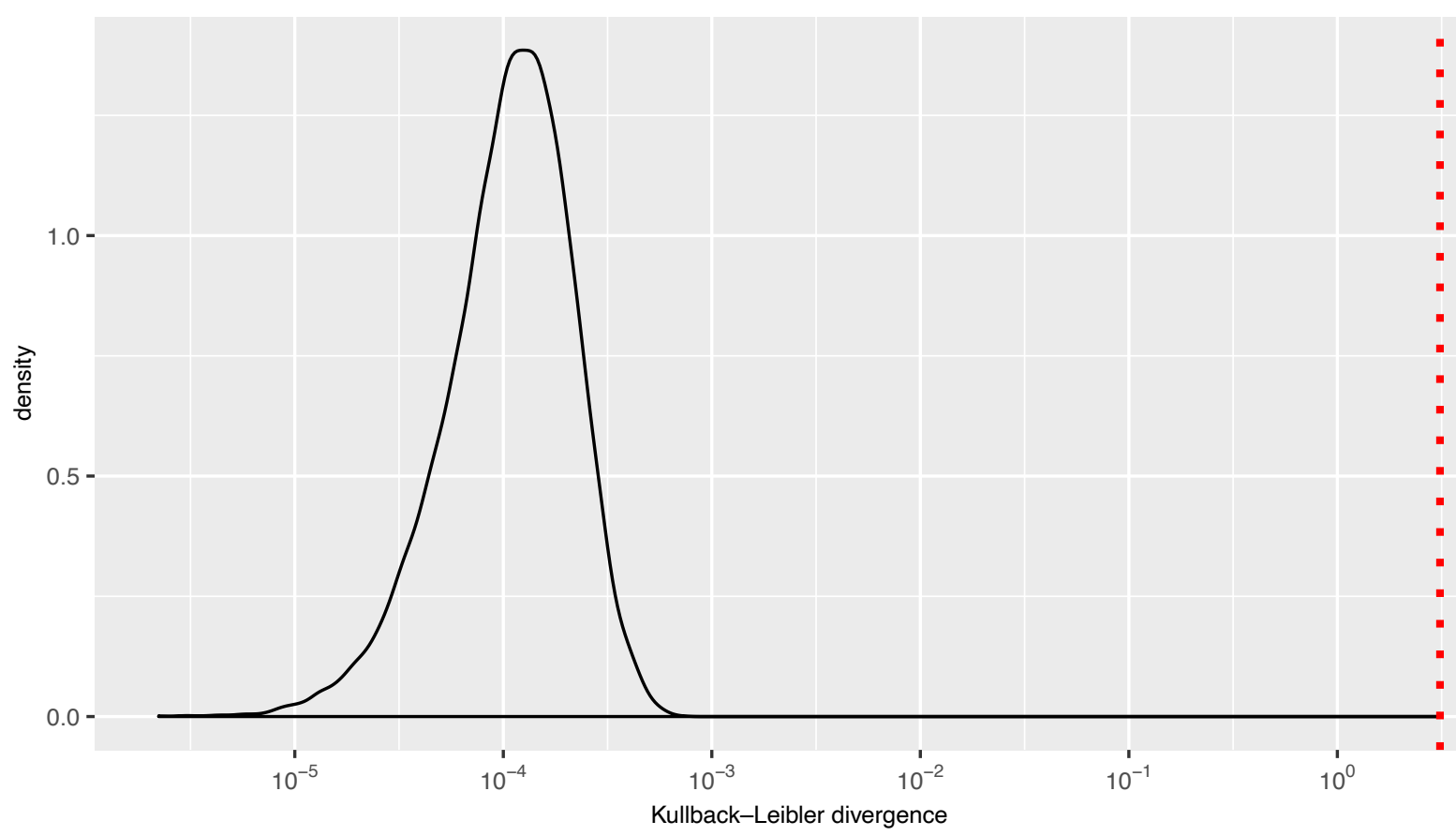

Figure 5. Kullback-Leibler divergence between the probability density functions of the information weights of younger and older adults. The dotted red line indicates the Kullback-Leibler divergence observed between the information weights of the younger and older adults in the present study. The distribution in black can be used to assess divergence values that could occur by chance. The distribution was obtained by 10,000 iterations of calculating the divergence after shuffling the Age group vector. The x-axis is log scaled.

\section{SL and Cognitive ability}

Figure 6 provides an overview of the magnitudes of the correlation values between SL as measured by CHPC and CRV by the end of the experiment, and all cognitive assessments conducted. The dendrogram is the result of hierarchical clustering of these magnitudes. Supplement S2 contains the full correlation matrix. 


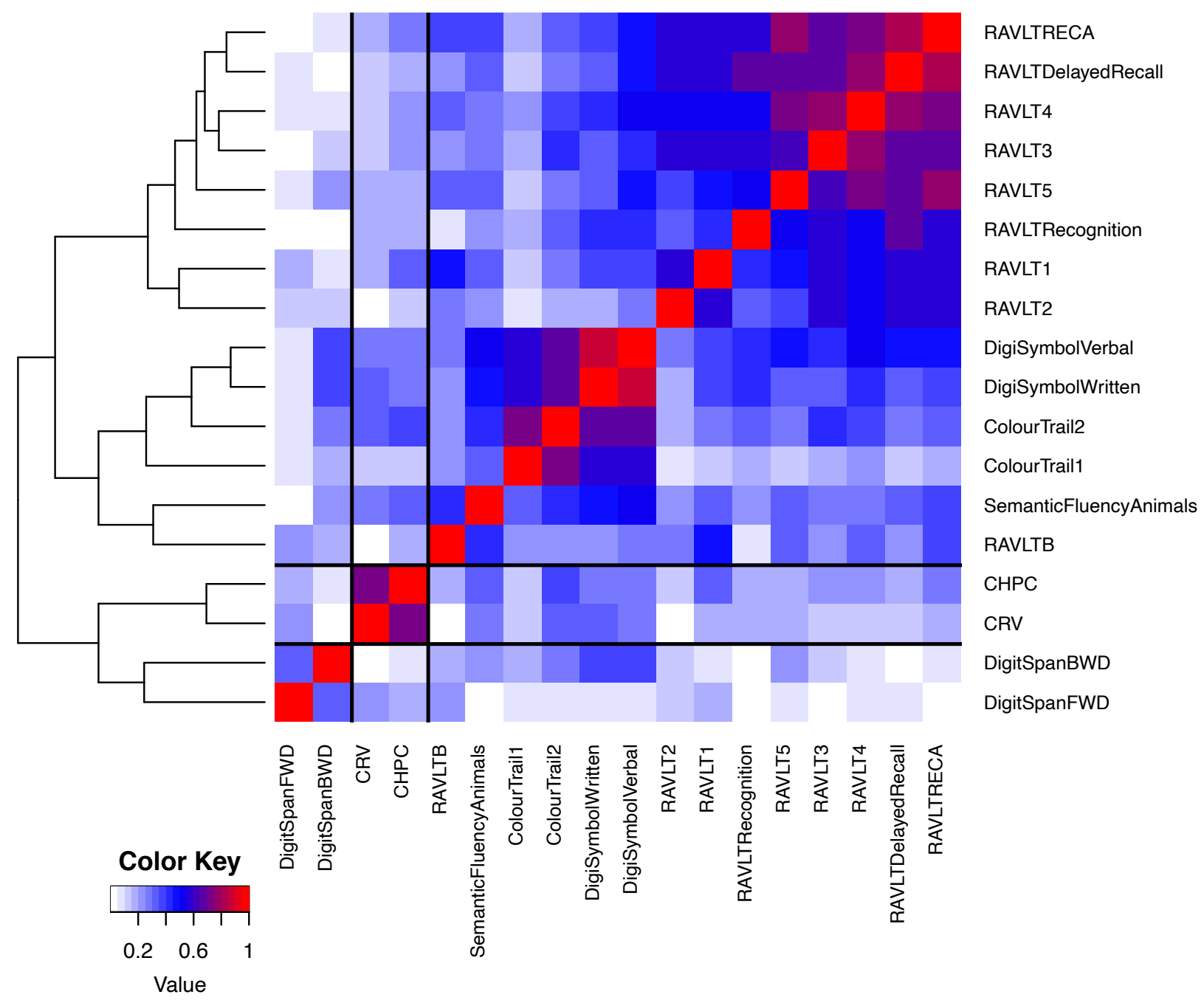

Figure 6: Hierarchical clustering of the magnitudes of the correlation coefficients of SL and all cognitive assessments. Even though both digit span tests are clustered the closest to CRV and CHPC, a stepwise regression revealed that RAVLT1 and DigitSymbolWritten carry the most predictive value for SL. The black lines highlight the cells related to CRV and CHPC.

Figure 6 shows that SL and most cognitive assessments tend to be clustered in two distinct groups of measurement. This, combined with the overall low correlations (all $r<.33$, see Supplement S2), points towards SL being distinct to the construct targeted by most cognitive assessment tests. However, this does not exclude the possibility that there are individual 
cognitive assessments that relate to SL. To address this as well as the small participants-topredictors ratio, a stepwise regression (both-ways, $\triangle B I C$ penalty term) was performed to reveal the best predictors for CHPC and CRV. For CHPC, RAVLT1 was the only remaining predictor, and for CRV, the DigitSymbolWritten test was the only surviving predictor.

Consequently, we deployed linear Bayesian mixed effects models predicting CRV and CHPC scores. The models were provided with a fixed factor for Age, Trial, as well as the RAVLT1 and DigitSymbolWritten scores. All interaction terms were fully parameterized, with the exception of RAVLT1 and DigitSymbolWritten interaction terms, as they are of no interest to the present design. We found that for both cognitive assessments, Trial $x \operatorname{RAVLT1}\left(\beta=1.01, E E_{\beta}=\right.$ $\left..09, \operatorname{Odds}(\beta>0)=>9999^{*}\right)$ and Trial $x$ DigitSymbolWritten $\left(\beta=.74, E E_{\beta}=.10\right.$, Odds $(\beta>0)$ $\left.=>9999^{*}\right)$, larger scores predicted steeper statistical learning trajectories. Furthermore, the Trial $x$ RAVLT1 $x$ OlderAdult $\left(\beta=.78, E E_{\beta}=.12, \operatorname{Odd}(\beta>0)=>9999^{*}\right)$ as well as Trial $x$ DSW $x$ OlderAdult $\left(\beta=.40, E E_{\beta}=.15, \operatorname{Odds}(\beta>0)=231.26^{*}\right)$ interaction terms showed that these effects are stronger in older adults compared to younger. This can also be seen in Figure 7 in the larger difference between the two colored lines in older adults compared to younger adults (see Supplement S2 for the full models). 

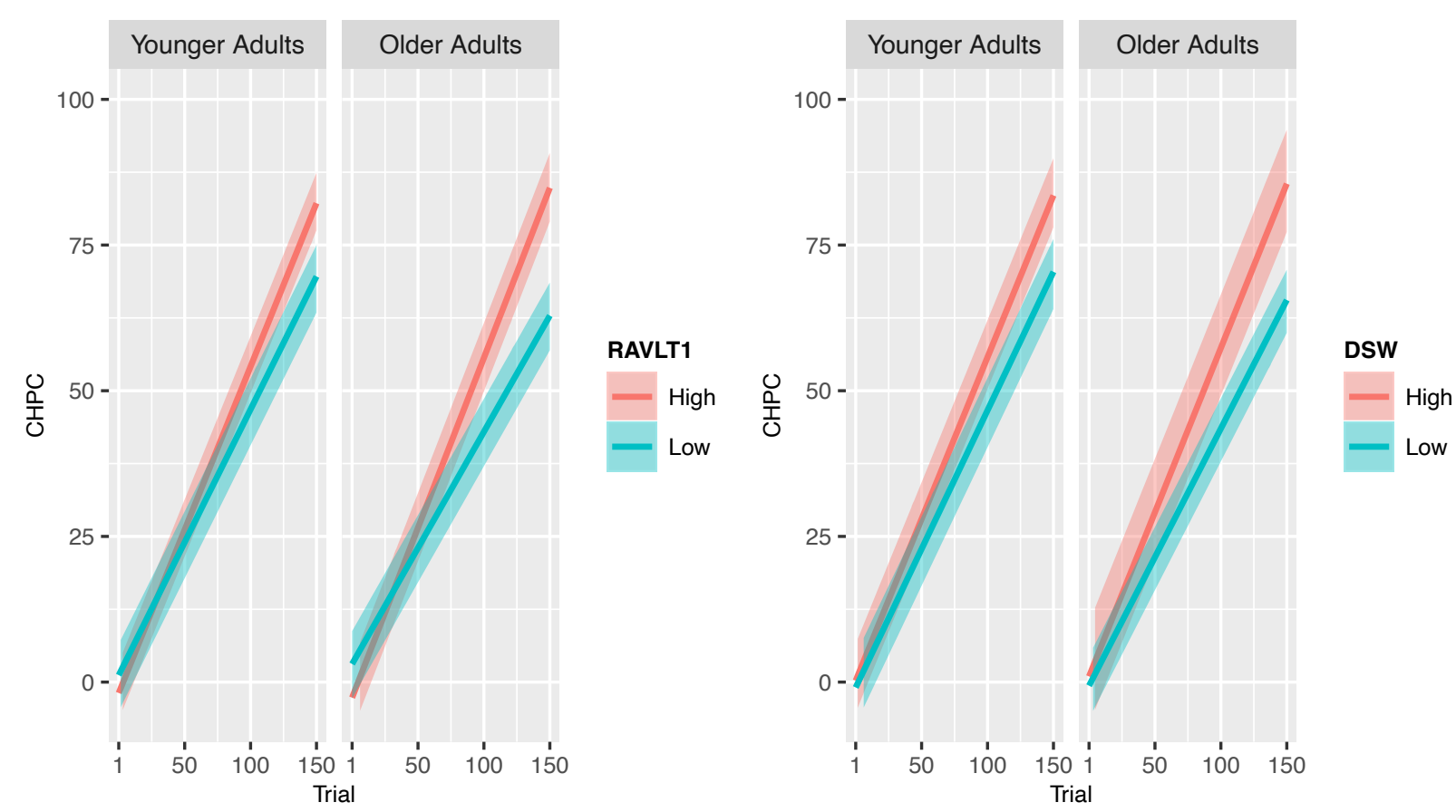

Figure 7. Marginal effects plots of Age, SL, and cognitive assessment scores. Both age groups show higher predicted CHPC values with high RAVLT1 (2.14) and high DSW (2.64, red lines) compared to low RAVLT1 (2.35) and low DSW scores (-2.08, blue lines). The larger distances between the red and the blue lines in older adults compared to the younger adults visualises the three-way interaction. The bands represent $95 \%$ CIs.

\section{Discussion}

We investigated differences in SL trajectories between younger and older adults, and the extent to which both groups learned the underlying statistical structure in the task. Both age groups showed similar learning trajectories of the most likely next event when the transition was likely (high certainty). When it came to dealing with less certain transitional probabilities, learning trajectories diverged between age groups. To explain these findings, we tested three cognitive models. We found that younger and older adults utilize similar strategies, but younger adults are more willing to change their behavior by placing strong weight on negative observations during their decision-making process. In addition, scores on some traditional 
cognitive assessments were found to mediate performance. This effect was stronger in older adults but did not explain the age-related response pattern in the present task, and may only be indicative of task-specific demands. The main contribution of this study is demonstrating agerelated differences in statistical learning which can be modelled through systematic shifts in information weights when sampling information.

\section{SL Performance and Age}

Many SL paradigms suffer from overall low performance (Siegelman, Bogaerts, \& Frost, 2017). Following previous suggestions (Herff, Nur, et al., 2019), we deployed a large number of trials and multi-modal stimuli, and found clear signs of learning in the majority of participants in both age groups. Overall, more young adults learned the most likely next event (CHPC) and approximated ideal performance compared to older adults. This is in line with previous studies that also showed an age-related decline in SL of probabilistic stimuli (Curran, 1997; Feeney et al., 2002; J. H. Howard \& Howard, 1997). Furthermore, more older adults failed to learn that immediate state repetitions (CRV) were impossible. This is an interesting observation that requires further exploration in the future, as presently CRV did not provide enough variability to be effectively modelled and the analysis focused on CHPC instead. Trial-wise analysis of CHPC revealed that younger adults show more high-probability responses initially, but the learning trajectories over time are comparable between the groups. This could be indicative of a more conservative strategy deployed by older adults initially, such as a stronger 'prior' inclination towards equiprobable responses in the beginning. Participants' behavior in light of likely and unlikely transitions reveal further insight.

\section{Certainty States}


In any probabilistic scenario, it is important to consider that the relationship between the probability of an outcome and an individual's predictions or decisions may not be a linear one. Indeed, examples of where observed probabilities and the resulting predictions or decisions have a distinctly non-linear relationship are well-documented (see Barberis, 2013 for a review). In the present paradigm, we were able to observe younger and older adults' behavior when dealing with likely or unlikely transitions, as the present paradigm contains both low- and high-certainty states. Here, we consider that low and high certainty may function as a proxy to task difficulty. This is because it is possible that probabilities of unlikely transitions are more difficult to extract, compared to likely transitions. Indeed, the present results suggest that learning of likely transitions is faster compared to unlikely transitions. However, if transitional probabilities only impacted task difficulty, then the prior literature would suggest that younger adults should outperform older adults on low-certainty states (Curran, 1997; Feeney et al., 2002; D. V. Howard et al., 2004; J. H. Howard \& Howard, 1997; Palmer \& Mattys, 2016). We did not observe this pattern in the present data. As a result, we conclude that transitional likelihood cannot be used as a direct proxy to task difficulty. That is not to say that difficulty does not vary with transitional likelihood, as the current design cannot exclude this possibility. However, the present results strongly indicate that transitional likelihood has other profound impacts on learning, beyond a potential impact on task difficulty.

Within the high-certainty states, learning trajectories between the two age groups were not significantly different from one another. However, when faced with less certain transitional probabilities, the response pattern in older adults stayed relatively constant and close to the actual underlying transitional probabilities throughout the experiment. Conversely, younger adults showed an initial strong tendency towards the most likely event, followed by a rapid decay 
in their likelihood of responding with the next most likely state (see Figure 3). At first glance, this observation is somewhat startling. Why should younger adults drift away from the true underlying probability, when they were perfectly capable of identifying it in the beginning? A key difference between the likely and unlikely transitions is that for unlikely transitions, a correct prediction (e.g., 'B' will follow 'A') may often not be realized, even though it may be the most likely transition (e.g., of all options ' $\mathrm{B}$ ' is the most likely one to follow 'A'). If younger adults showed a strong adverse reaction (e.g., frustration) to negative observations (' $\mathrm{B}$ ' did not follow 'A', despite the perception that ' $\mathrm{B}$ ' is the most likely), then this would conceptually capture this pattern of results. We will return to this point with a more formal explanation when discussing the results of the Information Weights model. This is because the way participants react to various degrees of certainty can be indicative of the underlying learning mechanisms used. Here, we tested three cognitive models to further explore the learning mechanisms underlying SL, as well as age-related differences.

\section{Learning Mechanisms}

We found evidence that both age groups draw information from the continuous sequence, rather than only from the last time they provided a response specific to the current state. That is, neither age group utilizes a Win-Stay, Lose-Shift Strategy. This is an important observation, as reliance on Win-Stay, Lose-Shift would have suggested that participants were not extracting the full underlying TP matrix, but instead only rely on a simple response heuristic to perform the SL task. Theoretically, many probabilistic SL tasks that provide explicit or implicit feedback could be performed reasonably well with a Win-Stay, Lose-Shift strategy (e.g., Matsen \& Nowak, 2004). If a SL task can theoretically be achieved with strategies that do not extract the underlying statistical dependencies, then additional steps need to be taken when interpreting the ability to 
perform a SL task as evidence that the learners extracted the underlying TP matrix. The fact that both groups did not utilize a Win-Stay, Lose-Shift strategy lends strength to the present paradigm and the results.

Delta-rule learning describes the results within both age groups well. By adjusting their behavior more strongly the further their own beliefs differ from the actual underlying probabilities, participants were better able to perform the task. As predicted, younger adults do this to a greater extent than older adults. The second hypothesis about delta-rule learning was also confirmed: participants were more willing to adjust their behavior at the higher end of the probability spectrum. Based on present results, delta-rule learning could be a crucial mechanism involved in statistical learning, and it could explain some of the age-related differences observed. However, the hypothesized interaction in delta-rule learning between transitional likelihood and age was not observed. As a result, age-related differences in delta-rule learning cannot explain the age-related differences observed in the low-certainty responses discussed in the previous section. The information weights model, on the other hand, can.

There is ample evidence that correct predictions are intimately tied with internally generated rewards (Fiser, Berkes, Orbán, \& Lengyel, 2010), which increase the probability of the same prediction in the future, similar to a Bayesian observer. However, the decrease in probability caused by a negative observation (' $\mathrm{B}$ ' does not follow ' $A$ ') may not be identical to the increase in probability caused by a positive observation ('B' does follow 'A'). With the data collected here, we were able to calculate the weights that younger and older adults attach to positive and negative transitional observations. We find that information weights offer a parsimonious mechanistic description that captures the present results well. As hypothesized, younger adults attached larger weights to both types of observations compared to older adults, 
which could explain why younger adults initially show faster behavioral changes. Most importantly, younger adults strongly weight the information of negative observations over positive ones when it comes to formulating future predictions. Older adults also rely on negative information more than on positive, but to a substantially lesser extent than younger participants. As a result, our second hypothesis about information weights is supported. It is important to note that older participants here appear close to equi-weighting for positive and negative observations.

Overweighting negative observations, as younger participants did, appears sensible from an evolutionary perspective, as it allows rapid discarding of impossible or unlikely - and therefore unreliable - outcomes. However, it would also lead to a greater shift away from the true underlying transitional probabilities. The decrease over time of high-probability choices in low-certainty states in the present study could be an example of this possibility. Interestingly, the lower but more balanced weights in older adults, in the long run, would yield more accurate yet slower behavioral changes. This fits the general observation that older adults weight accuracy over speed (Forstmann et al., 2011; Salthouse, 1979). The information weights perspective also integrates well with previous findings. Nassar et al. (2016) found large behavioral adjustments to relatively minor predictions errors in younger adults, but not older adults. This observation could be well-described by younger adults placing larger weights on negative observations, as the present information weights model revealed. A potential explanation for the age-related shift in information weights may be provided by socioemotional selectivity theory.

Socioemotional selectivity theory posits that goal-directed behavior is strongly influenced by an individual's perspective on time (Carstensen, 1992, 1995; Carstensen, Fung, \& Charles, 2003). Specifically, when time is perceived as open-ended, expensive and potentially risky longterm goals are considered. However, when time is perceived as being limited, greater importance 
is put on the present, for example, by prioritizing emotional wellbeing and stability. As age progresses, individuals tend to perceive time as passing faster, often paired with an increasing confrontation with one's own mortality. In the current study, the balanced information weights utilized by the older adults result in a slower change of behavior that is much less prone to dramatic shifts in behavior, and would eventually arrive at a stable homeostatic state with a response distribution that mirrors the precise underlying transitional probabilities. As behavioral change and inaccurate decisions are more expensive for individuals that perceive time as 'running-out', the balanced approach to weighting incoming information would be the most rational choice for older individuals, rather than deploying a weighting that prioritizes quick yet imprecise adaptation (as embraced by the younger adults).

Establishing individuals' information weights could be a useful tool for customizing and optimizing learning. Specifically, it seems that as age progresses, positive observations ('B' follows 'A') become more important for learning than negative observations. This finding could be relevant for an aging workforce that is required to adapt and learn new skills (WHO, 2015, 2017). However, it is important to note that the present study cannot distinguish 'age' from 'education' because the between-subjects nature of the design means that age-related differences may be a feature of aging, or a result of different upbringings between the two generations. In both cases, the information weights perspective may be useful as awareness of information weights may help in understanding the judgements made by oneself and others. It is apparent in the present results that different information weights can lead to different decisions at different timepoints. As a result, expressing decisions as a function of the information weights may help in bridging opposing judgements. Furthermore, exploring an information weights perspective could also be useful in deepening our understanding of mental disorders that can be understood as 
information filters (e.g., depression, see Gaddy \& Ingram, 2014). For example, depression could be characterized as elevated negative - or reduced positive - information weights. This question represents a promising area for future research.

\section{SL and Cognitive Ability}

To test whether results could also be explained by differences in cognitive function, we collected a battery of cognitive assessments from both age groups. Across younger and older adults, we found evidence that higher cognitive assessment scores predict steeper learning trajectories. Importantly, this effect was exacerbated in older adults. Specifically, whereas older adults with high cognitive assessment scores show similar SL performance compared to young adults with high cognitive assessment scores, older adults with low cognitive assessment scores show lower SL performance compared to younger adults with matched scores. A possible explanation could be that low cognitive assessment scores in older adults may be indicative of age-related cognitive decline that affects various functions in the brain, whereas low scores in younger adults are less likely to be indicative of functional impairments. The multi-modal paradigm may have reduced the cognitive demand of the task, but it also required visual-audio coordination, which in turn might pose a challenge, as perceptual processes decline with age. If this is the case, then this could also be a contributing factor explaining why the cognitive assessment scores were stronger predictors of SL in older adults.

Of the large number of cognitive tests deployed, the two most promising predictors of SL were the RAVLT 1 as well as the Digit Symbol (written) Modality test. This makes intuitive sense, as the Digit Symbol Modality test was designed to capture associative learning, and the RAVLT tests auditory memory. Clustering based on the correlation magnitudes and overall low correlations $(r=.33)$ further suggest that SL ability and traditional cognitive assessments most 
likely target different underlying constructs and any predictive information observed may be due to task-specific requirements (e.g. auditory tracking) instead of a direct influence of cognitive ability on SL (Feldman et al., 1995; Herff, Nur, et al., 2019; Kaufman et al., 2010; Siegelman, Bogaerts, \& Frost, 2017). This is in line with previous literature that suggests that SL and general cognitive function are largely independent (Feldman, Kerr, and Streissguth, 1995; Kaufman et al. 2010; Siegelman, Bogaerts, and Frost, 2017). Based on the present results, it seems unlikely that differences in cognitive function are what drives the age-related differences in SL observed here.

\section{Conclusion}

The paradigm deployed here tracked learning trajectories and revealed differences between younger and older adults in SL when it comes to dealing with uncertainty. A possible explanation was found in the form of age-dependent differences in information weighting, in which younger adults more readily adjust their behavior, but also weight negative observations (e.g., 'B' does not follow 'A') more strongly than positive observations (e.g., 'B' does follow 'A') compared to older adults. The weights deployed by younger adults favor rapid behavioral adaptation, whereas the weights used by older adults favor more precise behavioral adaptation over time. We hope that future research using this paradigm will provide precise estimates of individuals' information weighting of positive and negative predictive outcomes. 


\section{References}

Agres, K., Abdallah, S., \& Pearce, M. T. (2018). Information-theoretic properties of auditory sequences dynamically influence expectation and memory. Cognitive science, 42(1), 4376. doi:10.1111/cogs.12477

Aizenstein, H. J., Butters, M. A., Clark, K. A., Figurski, J. L., Stenger, V. A., Nebes, R. D., . . Carter, C. S. (2006). Prefrontal and striatal activation in elderly subjects during concurrent implicit and explicit sequence learning. Neurobiology of Aging, 27(5), 741751. doi:10.1016/j.neurobiolaging.2005.03.017

Barascud, N., Pearce, M. T., Griffiths, T. D., Friston, K. J., \& Chait, M. (2016). Brain responses in humans reveal ideal observer-like sensitivity to complex acoustic patterns.

Proceedings of the National Academy of Sciences, 113(5), E616-E625. doi:10.1073/pnas.1508523113

Barberis, N. C. (2013). Thirty years of prospect theory in economics: A review and assessment. Journal of Economic Perspectives, 27(1), 173-196. doi:10.1257/jep.27.1.173

Barr, D. J., Levy, R., Scheepers, C., \& Tily, H. J. (2013). Random effects structure for confirmatory hypothesis testing: Keep it maximal. Journal of Memory and Language, 68(3), 255-278. doi:10.1016/j.jml.2012.11.001

Bürkner, P. (2017). Brms: An r package for bayesian multilevel models using stan. Journal of Statistical Software, 80(1), 1-28. doi:10.18637/jss.v080.i01

Bürkner, P. (2018). Advanced bayesian multilevel modeling with the r package brms. arXiv preprint arXiv:1705.11123, 10(1). doi:10.32614/RJ-2018-017

Carstensen, L. L. (1992). Social and emotional patterns in adulthood: Support for socioemotional selectivity theory. Psychology and Aging, 7(3), 331-338. doi:10.1037//0882-7974.7.3.331 
Carstensen, L. L. (1995). Evidence for a life-span theory of socioemotional selectivity. Current Directions in Psychological Science, 4(5), 151-156. doi:10.1111/1467-8721.ep11512261

Carstensen, L. L., Fung, H. H., \& Charles, S. T. (2003). Socioemotional selectivity theory and the regulation of emotion in the second half of life. Motivation and Emotion, 27(2), 103 123. doi:10.1023/A:1024569803230

Cherry, K. E., \& Stadler, M. E. (1995). Implicit learning of a nonverbal sequence in younger and older adults. Psychology and Aging, 10(3), 379-394. doi:10.1037/0882-7974.10.3.379

Creel, S. C., Newport, E. L., \& Aslin, R. N. (2004). Distant melodies: Statistical learning of nonadjacent dependencies in tone sequences. Journal of Experimental Psychology: Learning, Memory, and Cognition, 30(5), 1119-1130. doi:10.1037/0278-7393.30.5.1119

Curran, T. (1997). Effects of aging on implicit sequence learning: Accounting for sequence structure and explicit knowledge. Psychological Research, 60(1-2), 24-41. doi:10.1007/BF00419678

D'Elia, L., Satz, P., Uchiyama, C. L., \& White, T. (1996). Color trails test: Ctt: Psychological Assessment Resources Odessa, FL.

Daltrozzo, J., \& Conway, C. M. (2014). Neurocognitive mechanisms of statistical-sequential learning: What do event-related potentials tell us? Frontiers in Human Neuroscience, 8, 437. doi:10.3389/fnhum.2014.00437

Eppinger, B., \& Kray, J. (2011). To choose or to avoid: Age differences in learning from positive and negative feedback. Journal of Cognitive Neuroscience, 23(1), 41-52. doi:10.1162/jocn.2009.21364. 
Feeney, J. J., Howard, J. H., \& Howard, D. V. (2002). Implicit learning of higher order sequences in middle age. Psychology and Aging, 17(2), 351-355. doi:10.1037/08827974.17.2.351

Feldman, J., Kerr, B., \& Streissguth, A. P. (1995). Correlational analyses of procedural and declarative learning performance. Intelligence, 20(1), 87-114. doi:10.1016/01602896(95)90007-1

Feng, L., Lim, W.-S., Chong, M.-S., Lee, T.-S., Gao, Q., Nyunt, M., . . Ng, T.-P. (2017). Depressive symptoms increase the risk of mild neurocognitive disorders among elderly chinese. The Journal of Nutrition, Health \& Aging, 21(2), 161-164. doi:10.1007/s12603016-0765-3.

Ferdinand, N. K., \& Kray, J. (2013). Age-related changes in processing positive and negative feedback: Is there a positivity effect for older adults? Biological Psychology, 94(2), 235241. doi:10.1016/j.biopsycho.2013.07.006

Fiser, J., Berkes, P., Orbán, G., \& Lengyel, M. (2010). Statistically optimal perception and learning: From behavior to neural representations. Trends in Cognitive Sciences, 14(3), 119-130. doi:10.1016/j.tics.2010.01.003

Forstmann, B. U., Tittgemeyer, M., Wagenmakers, E. J., Derrfuss, J., Imperati, D., \& Brown, S. (2011). The speed-accuracy tradeoff in the elderly brain: A structural model-based approach. Journal of Neuroscience, 31(47), 17242-17249. doi:10.1523/JNEUROSCI.0309-11.2011

Frensch, P. A., \& Miner, C. S. (1994). Effects of presentation rate and individual differences in short-term memory capacity on an indirect measure of serial learning. Memory \& Cognition, 22(1), 95-110. doi:10.3758/BF03202765 
Gaddy, M. A., \& Ingram, R. E. (2014). A meta-analytic review of mood-congruent implicit memory in depressed mood. Clinical Psychology Review, 34(5), 402-416. doi:10.1016/j.cpr.2014.06.001

Gelman, A., Stern, H. S., Carlin, J. B., Dunson, D. B., Vehtari, A., \& Rubin, D. B. (2013). Bayesian data analysis: Chapman and Hall/CRC.

Greve, A., Cooper, E. E., Kaula, A., Anderson, M. C., \& Henson, R. (2017). Does prediction error drive one-shot declarative learning? Journal of Memory and Language, 94, 149165. doi:10.1016/j.jml.2016.11.001

Herff, S. A., Nur, A., Lee, J., Lee, T., \& Agres, K. (2019). Statistical learning ability as a measure of cognitive function. Paper presented at Cognitive Science, Montreal, Canada, 24-27 July. doi:10.31234/osf.io/u4ry6

Herff, S. A., Zhen, S., Yu, R., \& Agres, K. R. (2019). Age-dependent statistical learning trajectories reveal differences in information weighting. Psyarxiv. doi:10.31234/osf.io/kuy6p

Hinault, T., Lemaire, P., \& Touron, D. (2017). Strategy combination during execution of memory strategies in young and older adults. Memory, 25(5), 619-625. doi:10.1080/09658211.2016.1200626

Howard, D. V., \& Howard, J. H. (1989). Age differences in learning serial patterns: Direct versus indirect measures. Psychology and Aging, 4(3), 357-364. doi:10.1037/08827974.4.3.357

Howard, D. V., \& Howard, J. H. (1992). Adult age differences in the rate of learning serial patterns: Evidence from direct and indirect tests. Psychology and Aging, 7(2), 232-241. doi:10.1037/0882-7974.7.2.232 
Howard, D. V., Howard, J. H., Japikse, K., DiYanni, C., Thompson, A., \& Somberg, R. (2004). Implicit sequence learning: Effects of level of structure, adult age, and extended practice. Psychology and Aging, 19(1), 79-92. doi:10.1037/0882-7974.19.1.79

Howard, J. H., \& Howard, D. V. (1997). Age differences in implicit learning of higher order dependencies in serial patterns. Psychology and Aging, 12(4), 634-656. doi:10.1037/0882-7974.12.4.634

Kaufman, S. B., DeYoung, C. G., Gray, J. R., Jiménez, L., Brown, J., \& Mackintosh, N. (2010). Implicit learning as an ability. Cognition, 116(3), 321-340. doi:10.1016/j.cognition.2010.05.011

Kirkham, N. Z., Slemmer, J. A., \& Johnson, S. P. (2002). Visual statistical learning in infancy: Evidence for a domain general learning mechanism. Cognition, 83(2), B35-B42. doi:10.1016/S0010-0277(02)00004-5

Krogh, L., Vlach, H., \& Johnson, S. P. (2013). Statistical learning across development: Flexible yet constrained. Frontiers in Psychology, 3, 598. doi:10.3389/fpsyg.2012.00598

Löckenhoff, C. E., \& Carstensen, L. L. (2007). Aging, emotion, and health-related decision strategies: Motivational manipulations can reduce age differences. Psychology and Aging, 22(1), 134-146. doi:10.1037/0882-7974.22.1.134

Mata, R., von Helversen, B., \& Rieskamp, J. (2010). Learning to choose: Cognitive aging and strategy selection learning in decision making. Psychology and Aging, 25(2), 299-309. doi:10.1037/a0018923

Matsen, F. A., \& Nowak, M. A. (2004). Win-stay, lose-shift in language learning from peers. Proceedings of the National Academy of Sciences, 101(52), 18053-18057. doi:10.1073/pnas.0406608102 
Milne, A. J., \& Herff, S. A. (2020). The perceptual relevance of balance, evenness, and entropy in musical rhythms. Cognition. doi:10.1016/j.cognition.2020.104233

Misyak, J. B., Christiansen, M. H., \& Tomblin, J. B. (2010). On-line individual differences in statistical learning predict language processing. Frontiers in Psychology, 1, 31 . doi:10.3389/fpsyg.2010.00031

Moldwin, T., Schwartz, O., \& Sussman, E. S. (2017). Statistical learning of melodic patterns influences the brain's response to wrong notes. Journal of Cognitive Neuroscience, 29(12),2114-2122.doi:10.1162/jocn_a_01181

Nassar, M. R., Bruckner, R., Gold, J. I., Li, S. C., Heekeren, H. R., \& Eppinger, B. (2016). Age differences in learning emerge from an insufficient representation of uncertainty in older adults. Nature Communications, 7(1), 1-13. doi:10.1038/ncomms11609

Nowak, M. A., \& Sigmund, K. (1993). A strategy of win-stay, lose-shift that outperforms tit-fortat in the prisoner's dilemma game. Nature, 364(6432), 56-58. doi:10.1038/364056a0

Palmer, S. D., Hutson, J., \& Mattys, S. L. (2018). Statistical learning for speech segmentation: Age-related changes and underlying mechanisms. Psychology and Aging, 33(7), 10351044. doi:10.1037/pag0000292

Palmer, S. D., \& Mattys, S. L. (2016). Speech segmentation by statistical learning is supported by domain-general processes within working memory. Quarterly Journal of Experimental Psychology, 69(12), 2390-2401. doi:10.1080/17470218.2015.1112825

Pérez-González, D., \& Malmierca, M. S. (2014). Adaptation in the auditory system: An overview. Frontiers in integrative neuroscience, 8(19), 1-10.

doi:10.3389/fnint.2014.00019 
Randolph, C., Braun, A. R., Goldberg, T. E., \& Chase, T. N. (1993). Semantic fluency in alzheimer's, parkinson's, and huntington's disease: Dissociation of storage and retrieval failures. Neuropsychology, 7(1), 82-88. doi:10.1037/0894-4105.7.1.82

Rescorla, R., \& Wagner, A. R. (1972). A theory of pavlovian conditioning: Variations in the effectiveness of reinforcement and nonreinforcement. Classical conditioning II: Current research and theory, 2, 64-99.

Rey, A. (1958). L'examenclinique en psychologie [the psychological examination]. Paris: Presses Universitaires de France.

Roseberry, S., Richie, R., Hirsh-Pasek, K., Golinkoff, R. M., \& Shipley, T. F. (2011). Babies catch a break: 7-to 9-month-olds track statistical probabilities in continuous dynamic events. Psychological Science, 22(11), 1422-1424. doi:10.1177/0956797611422074

Saffran, J. R., Aslin, R. N., \& Newport, E. L. (1996). Statistical learning by 8-month-old infants. Science, 274(5294), 1926-1928. doi:10.1126/Science.274.5294.1926

Saffran, J. R., \& Kirkham, N. Z. (2018). Infant statistical learning. Annual Review of Psychology, 69, 181-203. doi:10.1146/annurev-psych-122216-011805

Salthouse, T. A. (1979). Adult age and the speed-accuracy trade-off. Ergonomics, 22(7), 811821. doi:10.1080/00140137908924659

Salthouse, T. A., McGuthry, K. E., \& Hambrick, D. Z. (1999). A framework for analyzing and interpreting differential aging patterns: Application to three measures of implicit learning. Aging, Neuropsychology, and Cognition, 6(1), 1-18. doi:10.1076/anec.6.1.1.789

Schirda, B., Valentine, T. R., Aldao, A., \& Prakash, R. S. (2016). Age-related differences in emotion regulation strategies: Examining the role of contextual factors. Developmental Psychology, 52(9), 1370-1380. doi:10.1037/dev0000194. 
Shafir, S., Reich, T., Tsur, E., Erev, I., \& Lotem, A. (2008). Perceptual accuracy and conflicting effects of certainty on risk-taking behaviour. Nature, 453(7197), 917-920. doi:10.1038/nature06841

Siegelman, N., Bogaerts, L., Christiansen, M. H., \& Frost, R. (2017). Towards a theory of individual differences in statistical learning. Philosophical Transactions of the Royal Society B: Biological Sciences, 372(1711), 20160059. doi:10.1098/rstb.2016.0059

Siegelman, N., Bogaerts, L., \& Frost, R. (2017). Measuring individual differences in statistical learning: Current pitfalls and possible solutions. Behavior Research Methods, 49(2), 418432. doi:10.3758/s13428-016-0719-z

Siegelman, N., \& Frost, R. (2015). Statistical learning as an individual ability: Theoretical perspectives and empirical evidence. Journal of Memory and Language, 81, 105-120. doi:10.1016/j.jml.2015.02.001

Smith, A. (1982). Symbol digit modalities test: Western Psychological Services Los Angeles, CA.

Sohoglu, E., \& Chait, M. (2016). Detecting and representing predictable structure during auditory scene analysis. eLife, 5, e19113. doi:10.7554/eLife.19113

Tan, J., Tsakok, F. H., Ow, E. K., Lanskey, B., Lim, K. S. D., Goh, L. G., . . Foo, R. (2018). Study protocol for a randomized controlled trial of choral singing intervention to prevent cognitive decline in at-risk older adults living in the community. Frontiers in Aging Neuroscience, 10, 195. doi:10.3389/fnagi.2018.00195

Thorndike, E. L. (1898). Animal intelligence: An experimental study of the associative processes in animals. The Psychological Review: Monograph Supplements, , 2(4), i-109. doi:10.1037/h0092987 
WHO. (2015). World report on ageing and health. Retrieved from https://www.who.int/ageing/events/world-report-2015-launch/en/

WHO. (2017). Amendments to the staff regulations and staff rules. Retrieved from https://apps.who.int/gb/ebwha/pdf files/EB141/B141 11-en.pdf

Worthy, D. A., Hawthorne, M. J., \& Otto, A. R. (2013). Heterogeneity of strategy use in the iowa gambling task: A comparison of win-stay/lose-shift and reinforcement learning models. Psychonomic Bulletin \& Review, 20(2), 364-371.

Yu, C. H. (2018). Neuropsychological assessments training manual for assessors (T. Y. Qian \& S. J. Ching Eds. Version 3.1, Approved by: Heok, K. E., Feng, L. ed.). Singapore: Yong Loo Lin School of Medicine's Department of Psychological Medicine. 